\title{
Optimized and efficient deblurring through constraint conditional modelling
}

\author{
Ravikumar H C $\mathbf{C}^{1}$, Karthik $^{2}$ \\ ${ }^{1}$ Department of ECE, KSSEM, Bengaluru \& Asst. Prof., Department of ECE, Ghousia College of Engg, Ramanagaram, \\ VTU, India \\ ${ }^{2}$ Department of ECE, KSSEM, Bengaluru, VTU, India
}

\begin{tabular}{l} 
Article Info \\
\hline Article history: \\
Received Oct 14, 2020 \\
Revised Dec 7, 2020 \\
Accepted Dec 22, 2020 \\
\hline
\end{tabular}

\section{Keywords:}

Constraint conditional model

Convolution

Deblurring

Image restoring

\begin{abstract}
Image deburring technique refers to restoring an image from the degraded version named blurred. Blurring can be caused due to various phenomena such as optical system, motion blur and other phenomena. Moreover, to deblur the image it is essential to know the blurring process characteristics and it is one of the difficult task. In past several deblurring algorithm have been proposed to approximate the kernel blur, however they lack the efficiency and expensive to be applied for the real world scenario. In this paper, we have proposed a CCM (constraint conditional model) to deblur the image; it learns the direct mapping from the degraded to the absolute clean image. Moreover, the main aim of CCM is to restore the image in its original form, the best advantage of CCM is that it provides handsome tradeoff between the image quality and efficiency. Moreover, CCM is evaluated on the three different standard datasets by considering the different performance metrics and through the comparison analysis observation has made that CCM approach outperforms the other techniques.
\end{abstract}

This is an open access article under the CC BY-SA license.

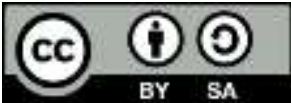

\section{Corresponding Author:}

Ravikumar H C

Department of Electronics and Communication Engineering

KSSEM, Bangalore 560109

Visvesvaraya Technological Universisty, Belagavi, Karnataka, India

Email: ravik_hc@rediffmail.com

\section{INTRODUCTION}

In past few decades, imaging science has seen a whole lot of revolution such as microscopy, medical imaging, astronomical imaging, consumer photography, moreover to support the application plenty of research has been carried out. These researches enable the image acquisition in higher speed, however, faster image aquisition methods leads to the degradation in image quality, and blur is one of the best examples. In general, blur is occurred due to the various scenarios such as if fast moving vehicle captured through the surveillance system leads to the blurrness in the image or video and this leads to difficulty in identifying the license plates. Other scenarios occur such as it is very difficult to stabilize the camera for the longer periods, mainly in the low lighting conditions; since it needs, the long exposure time which results in blurred images [1]. Moreover, also it is observed that the imaging system designed have single focus and other remains blur, other scenario such as long distance capturing or sometimes lens fails to focus on the entire available color on the given convergence point, this might result in the blurred image. Based on the above-discussed scenario the blur can be described as different types [2]. 
a) Object motion blur: This type of motion blur is caused due to the relative motion between the camera system and the object in scene at the given exposure time and it occurs during the exposure time [3].

b) Camera Shake Blur: This type of blur is induced through the camera motion at the exposure period and this is common in the dim light and mostly exist in handheld photography. This type of blur can be complex since the hand might move in the unexpected direction and might cause the rotation of camera out of plane or in plane [4].

c) Intrinsic Physical Blur: This type of blur exists due to the several reasons such as anti-aliasing filters, sensor resolution, lens aberration and light diffraction. For example, in an optical system, light rays' travels from one point converge at the same point on the focal plane and results in the sharp image, whereas in the real it is highly improbable that light rays travel parallel on the given optical axis.

d) Defocus Blur: Moreover, this is the most common types of blur and it is observed in the everyday photos we take such as selfies, for instance to capture the image for a novice photographer it is very difficult to focus the particular object. Similarly, when the camera has single lens then scene outside the field is on the blurry side.

Moreover, the above discussion shows the problem facing in capturing the images and since recent years hve seen the popularity of capturing the images and videos through the mobile and hand held cameras and most likely the blurry images are produced due to the several reasons discussed earlier in this section. Furthermore, the blur in any given image can restrict the human to resolve the scene detail; hence discarding the artifacts becomes the very critical process. Moreover, to get rid of such issue, deblurring was introduced which tries to restore the image, as in its original form, in past several researches have been taken for deblurring. Based on this research the deblurring process is classified as the non-blind deblurring and blind deblurring. Moreover, when the motion trajectories of camera are known then the estimating the image is known as the non-blind deblurring, when the information is missing then it is called blind deblurring. Generally, blind deblurring is difficult, as it requires the good amount of knowledge of blur and the desired image. Moreover, to gather good knowledge of blur kernel and the image still several researches is required, in past several researchers have proposed to deblur the image by retrieving the prior knowledge, some of them have been discussed in the next section i.e. literature survey, however these methods are either nonlinear or nonconvex.

Hence, in this paper we have proposed the novel methodology, which helps in deblurring the images, here we used 8 blur kernel and proposed the CCM (constraint conditional model) along with convergence operator that helps in restoring the images. We use CNN based filter for achieving the desired image, Moreover the contribution of this particular research work has been highlighted through the below points.

a) We propose constraint conditional model along with the convergence operator to restore the image.

b) CCM (constraint conditional model) achieves the marginally computational efficiency when compared to the other method.

c) CCM possesses various characteristics such as robust in nature, flexible and easy to adopt.

d) CCM is compared to the various state-of-art techniques in terms of two performance metrics named as PSNR and SSIM, CCM performs marginally better than the other does.

This research work has organized and parted into four distinctive section, first section describes the background of blurring and the importance of deblurring, objectives and the contribution of research work. Second section discuss the various techniques which have proposed in past along with their advantages and disadvantages. Third section represents constraint conditional model along with mathematical equation, algorithm and the formulation. Fourth section presents the Evaluation of CCM by considering the various dataset and various scenarios.

\section{LITERATURE SURVEY}

Nowadays, numerous works have shown the significance of edge data in the blur kernel estimation, for instance, to identify the edges orientation as well as the location. Moreover, a Gaussian edge detector is a sub-pixel difference utilized in as edges are assumed to be detectable regardless of whether the quality is not that much good [5]. The bilateral filter is included in explicit edge prediction, which is utilized in [6], and in order to create edges of the salient image the shock filter is utilized, however, these techniques act efficiently for little blur degradation and that technique are not efficient for the kernels, which are of large-scale. To upgrade the searching for an edge, applied the minimization of the approximate L0 gradient [7]. Motivated by this work, the numerous current methodologies utilized the L0 norm 1 to apply the sparsity on the gradient of the image and furthermore thought to be various types of specific feature highlights for the latent image of intermediate. Further inclusive an extra L0 constraint of sparsity on image intensity itself for deblurring content in an image [8]. For a few images, an observation that genes that the clean image is sparser as 
compared to the dark channel of the images which are not clear (blurred). At that point, the sparsity is forced on the channel which is dark to additionally improvise the estimation blur of the kernel. The prior of the dark channel is less likely to assist the kernel's estimation if it dominates of pixels that are bright from the image input [9]. Previous work is based on the specific method between clean one and blurred image, and priors of pre-set as for the blur estimation are proposed. Similarly, the content of the state-of-the-art strategy of deblurring has improved performance on the content in the image while behaving not so well on the blurred of genuinely apprehend in the against, while an inert image inappropriate channels have the fracture of statistical [8]. Meanwhile, the likely techniques may have exceptionally promising outcomes which can be a watch. However, they fail in the content image [9, 10]. Gaussian factors utilized a zero-mean mixture to approximate gradients of the natural image distribution or heavy-tailed [11]. Nevertheless, considering the adjacent pixel's statistical data during the estimation of the kernel may generate ringing artifacts in the reestablished image. This model of probability that is unified and is fitted for the distribution of image gradient as well as in which priors of spare depends on the basic of MAP architecture lean toward image which is not cleared over a clear image [12]. To face this problem together with an issue, proposed a powerful effective standardized sparsity prior (so-called (11/12) regularization) for stabilizing the process of the kernel. Straightforwardly utilized 10 regularization to oblige the estimation of the kernel, which ensures the accuracy as well as increase the evaluation capacity. In further research, used 10-12 joint regularizations in kernel's evaluation together with the restoration of the latent image to improve the robustness of the algorithm [13]. Because single images of sparsely represented can introduce constraints on information fidelity terms, utilized over-complete coefficients of sparse to encode a block of image and consequently tries these coefficients to the deblurring method. Joined the images that are sparse natural prior along with the increase and better group of over-complete of a dictionary, thereby systematically structure of excluding detrimental [14]. Utilized the 11 standard to show the frame lets sparsity in a multi-scale methodology that returns from course to fine goals [15].

Applied a low-position paradigm together with the weighted atomic standard minimization used to fit for the matrix of low-position [16]. This strategy removes details of edges that are too small and fine texture when keeping up the structures of blurred images dominant, making it reasonable and suitable for pictures with rich subtleties. Presented a viable and effective technique that utilizations data from the channel which are dark, but priors fail depends on a dark channel to keep as for images in which bright pixels and better play the main job [17]. To address this insufficiency, joined bright as well as dark channel priors, presenting the 'higher channel prior'. Numerous deblurring strategies that do not depend on exist of image priors. These methods based on the locally constructed salience and match features but do not achieve correct results when source images consist of considerable noise [18, 19]. However, efficiency properties and convergence of the referenced algorithm have not been very much adjusted balanced. Besides, a few specialists have considered remarkable edge extraction and determination to propose various viable strategies of deblurring. Most of the strategies of de-blurring focus on center around situations, for example, low enlightenment, content, and faces. These areas of domain-specific techniques require complex activities for the operation.

\section{PROPOSED METHODOLOGY}

In past several methodologies have been proposed for the de-blurring process discussed in previous section, however these methods have failed miserably. Moreover, some of the method does achieve in deblurring but they fail in learning the parameter and bad trade off. Hence, to get rid of such scenario, we have proposed Constraint conditional model that can help in achieving a de-blurred image. The main advantage over the existing model is that it learns all the parameters and achieves the better trade off in time, efficiency and quality. CCM model is described below.

\subsection{Preliminarie}

Let us consider any two vectors $I_{1}$ and $I_{2}$ and both vectors may represent image observed and the expected image along with the func as the sensing operator. Furthermore, the image formation can be given through the (1):

$$
\begin{aligned}
& I_{1}=\operatorname{func}\left(I_{2}\right) \\
& \text { func }\left(I_{2}\right)=\mathcal{O} \otimes I_{2}+\mathcal{P}
\end{aligned}
$$

Where $\mathcal{O}$ is the blur kernel and $\otimes$ indicates $2 \mathrm{D}$ convolution between the expected image and blur kernel. Moreover, the operator is linear so it can be written as: 


$$
I_{1}=\mathrm{MI}_{2}+\mathcal{P}
$$

M Is the deconvolution with $\mathcal{O}$.

\subsection{Image learning framework}

Moreover, in this subsection, we propose a framework for learning for the whole image through the convolutional Neural Network based on neighborhood model; this neighborhood model connects the node given in the square region. In order to construct the framework, we define the potential function:

$$
f\left(\mathbb{I}_{(k)}=f_{\mathbb{F}}\left(\mathbb{I}_{(\mathbb{c})} ; \Theta\right)=\prod_{\mathrm{m}=1}^{\mathbb{R}} \omega\left(\mathbb{K}_{\mathrm{m}}^{\mathbb{E}} \mathbb{I}_{(\mathbb{c})} ; \boldsymbol{\rho}_{\mathbb{m}}\right)\right.
$$

In the above equation, $\mathbb{K}_{\mathrm{m}}$ indicates the linear filter, $\boldsymbol{\rho}_{\mathrm{m}}$ is parameters, $\Theta$ is set of all parameters, $\mathbb{R}$ is number of experts and chosen based on the model quality. Hence overall framework is given through the (5):

$$
\mathbb{Q}_{\mathbb{F}}(\mathbb{I} ; \Theta)=Z(\Theta)^{-1} \sum_{\mathbb{C}=1}^{\mathbb{C}} \sum_{\mathbb{m}=1}^{\mathbb{R}} \Omega\left(\mathbb{K}_{\mathbb{m}}^{\mathbb{E}} \mathbb{I}_{(\mathfrak{C})} ; \boldsymbol{\rho}_{\mathrm{m}}\right)
$$

$\mathbb{K}_{\mathrm{m}}^{\mathbb{E}}$ is linear filter which indicates the vector space of given pixel value, further we normalize the equation. To achieve that we take $\log$ of the model defined through $\psi$ and rewrite the (6) as:

$$
\mathbb{Q}_{\mathbb{F}}(\mathbb{I} ; \Theta)=\mathbb{D}(\Theta)^{-1} \exp \left\{-\mathbb{T}_{\mathbb{F}}(\mathbb{I} ; \Theta)\right\}=\mathbb{D}(\Theta)^{-1} \exp \left\{\sum_{\mathbb{c}=1}^{\mathbb{c}} \sum_{\mathbb{m}=1}^{\mathbb{R}} \psi\left(\mathbb{K}_{\mathbb{m}}^{\mathbb{E}} \mathbb{I}_{(\mathbb{c})} ; \boldsymbol{\rho}_{\mathfrak{m}}\right)\right\},
$$

\subsection{Improvised image learning framework}

Moreover, in order to develop the specific framework, selection of function is crucial and denoted as $\Omega\left(\mathbb{L} ; \rho_{\mathbb{m}}\right)$, here $\mathbb{L}$ indicates the filter response. The (7) presents the framework from the above scenario:

$$
\Omega_{\mathbb{J}}(\mathbb{L} ; \boldsymbol{\rho}, \tau)=e^{-\boldsymbol{\rho} \sqrt{\tau+\mathbb{L}^{2}}}
$$

Since $\Omega$ can be scaled through filter norms, hence we set $\tau=$ one, furthermore we log the framework function and de function derivative with respect to $\mathbb{L}$ and $\rho$.

\subsection{Objective function}

The above section frames the Image Learning Framework, this captures the absolute natural scene and this framework allows the generic prior model for the low-level vision. Furthermore, the overall framework with the objective function is represented in the (8):

$$
0.5 \Gamma\left\|I_{1}-M I_{2}\right\|_{2}^{2}+\sum_{n=1}^{R} \omega_{n}\left(B_{n} I_{2}\right)
$$

In the objective equation, $I_{1}$ and $I_{2}$ is the observed image and desired image respectively, convolution operator is denoted by $M, B_{n}$ is $2 \mathrm{D}$ conv and $b_{n}$ is filter. Since above problem is constrained problem and it is optimized through the penalty function and it is indicated as $\omega_{n}$. In order to balance the data fidelity and regularization, positive scalar is used and denoted as $\lambda$, the positive scalar depends on parameter and task assigned. Considering the above problem several researcher have tried to deblur image, moreover they trained for model mapping, few researcher have tried to achieve the tradeoff between the image quality and time, the main issue with these method is that it needed the individual training for the subtask in deblurring and furthermore trade off needs to be achieved hence by considering these issue we design constraint conditional model for achieving the high tradeoff.

\subsection{Optimization with penalty function}

In (8) presents the objective function and as it is constrained problem it needs to be optimized; hence we use the penalty function to optimize the function. Due to the non-convexity of the function, the function has to be optimized and can be optimized through the below process. Let us consider any random process $(\mathbb{M}, \mathbb{N})$ under $\pi(m, \mathbb{m}, \mathbb{L})$ and the temperature temp is defined through the (9):

$$
\Omega_{t e m p}(\mathbb{m}, \mathbb{m}, \mathbb{L})=\frac{\exp (-\Omega *(\mathrm{~m}, \mathrm{~m} ; \mathbb{L}) / t e m p)}{\left.\iint \exp (-\Omega *(\mathrm{~m}, \mathbb{m} ; \mathbb{L}) / t e m p) d \mathbb{M} d \mathbb{N}\right)}
$$

Here CD (Conditional Distribution) of $\mathbb{M}$ while $\mathbb{N}=\mathbb{m}$ can be given as: 


$$
\pi_{t e m p}(\mathbb{M} \mid \mathbb{m})=\pi_{t e m p}(\mathbb{M}, \mathbb{m})\left(\pi_{t e m p}(\mathbb{M}, \mathbb{m}) d \mathbb{M}\right)^{-1}
$$

Later Markov Chain is constructed and can be formulated as $\{(\mathbb{M}(\mathbb{C}), \mathbb{N}(\mathbb{C})), \mathbb{C}=0,1,2 \ldots$.$\} is$ constructed through the below process:

Step 1: let's select any temperature sequence $\left\{\right.$ temp $\left._{\mathbb{C}}, \mathbb{C}=1, \ldots.\right\}$.

Step 2: Initialize chain with zero as its value.

Step 3: In case of each $\mathbb{C}=1,2$, generate $(\mathbb{M}(\mathbb{C}), \mathbb{N}(\mathbb{c}))$ from $(\mathbb{M}(\mathbb{C}-1), B(\mathbb{C}-1))$ through transistor probability $\pi_{\text {temp }}\left((\mathbb{M} \mid B(\mathbb{C}-1)) \pi_{\text {temp }}(\mathbb{\mathbb { C }} \mid \mathbb{M})\right.$.

\subsection{Dividing problem into sub-problem}

In (8) is considered as the objective function, later through the above equation we optimize, however the problem rises in further optimization. Optimization is highly improbable while there is non-linearity in penalty function, further optimization can be achieved through by using the convergence operator which is discussed later in the same section, COP (convergence operator) helps in dividing the problem into distinctive sub-problems. At first, we frame non-linear estimator, this can be derived from the non-convex regularization, by considering these scenarios this problem is considered as the approximation problem and later we relax the problem using convergence operator function which is formulated in the next section. Meanwhile the approximation problem is framed with the new objective and depicted in (11):

$$
\left.0.5 \Gamma\left\|I_{1}-M I_{2}\right\|_{2}^{2}+0.5 \varphi \| C O\right)-M \|_{2}^{2} \sum_{n=1}^{R} \omega_{n}
$$

In the above equation, $\varphi$ indicates the positive scalar and $M$ indicates the convolution operator; here the value of $\lambda$ depends on the value of positive scalar and convolution operator. $\mathrm{CO}$ is given variable which is used for forming the equality; further we optimize (11) through the iterative approach using $\mathrm{CO}$ variable and includes the desired image $I_{2}$; moreover (12) and (13) is formulated through the iterative optimization.

\subsection{Approximation through Convergence operator}

In the above equation, $\varphi^{e}$ increase along with each iteration and it results in ideal approximation in $I_{2}$; further we employ the partition mechanism and this parts the problem into convergence operator based and distributable convergence operator based. Here we use CCM to train the model since the problem can be identified as non-deterministic and non convex. Moreover, the CCM (constraint conditional model) is developed and formulated in the next section:

$$
\begin{aligned}
& C O_{e}=\underset{C O}{\operatorname{argmin}}\left(\varphi^{e} \frac{1}{2}\left\|C o-M^{e-1}\right\|_{2}^{2}+\sum_{n=1}^{R} \omega_{n}\left(B_{n} C O\right)\right) \\
& I_{2}=\underset{I_{2}}{\operatorname{argmin}}\left(\lambda\left\|I_{1}-M I_{2}\right\|_{2}^{2}+\varphi^{e}\left\|C O_{e}-I_{2}\right\|_{2}^{2}\right)
\end{aligned}
$$

\subsection{Constraint conditional model}

In (13) we observe that the particular equation is dependent convergence operator are very much independent from the restoration task; however, fidelity weight $\lambda$ and matrix $M$ are problem specific. Moreover, CCM approach is easily transferable and can be easily replaced through convergence operator that are embedded for optimization. Furthermore (12) constitutes the de-blur on given image; thus, applying CCM on (12) we get:

$$
C O_{e}:=\operatorname{COP}_{\Theta}\left(M^{e-1}, \varphi_{e}\right)
$$

COP is the Convergence operator and $\Theta$ indicates the model parameter and it includes the amount of filter used in the process. Further we train the model $C O P_{\Theta}$ and Fidelity weight through the given trained data. Moreover, (12) and (13) are two sub-problem, and both are trained using the convergence operator.

\subsubsection{CCM algorithm}

The above algorithm is designed for constraint conditional model, i.e. the whole process is summarized in systematic manner through the above algorithm. Moreover, in CCM the one task is dependent on the other task hence the network architecture should maintain the convergence property and to achieve that we design the distributable convergence operator in the next section:

Step1: start

Step2: Intialize $I^{0}=I_{1}$ 


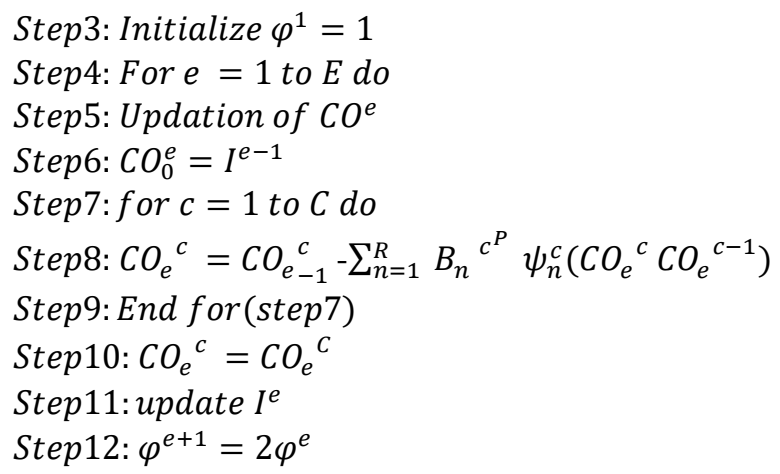

\subsection{DCOP (distributable convergance operator)}

In here we adopt the non-linear process named as multiple stage diffusion aka MSD for further optimization:

$$
\mathrm{CO}_{e}{ }^{c}=\mathrm{CO}_{e_{-1}}^{c}-\sum_{n=1}^{R} B_{n}{ }^{c^{P}} \psi_{n}^{c}\left(\mathrm{CO}_{e}{ }^{c} \mathrm{CO}_{e}{ }^{c-1}\right)
$$

Such that it should satisfy the below equation i.e. (16):

$$
\mathrm{CO}_{e}{ }^{o}=\mathrm{M}
$$

In (15), for initial stage and last stage we use $\psi_{n}^{c}$ as trainable parameter, $B_{n}{ }^{c}$ is noted as filter and c indicates stage index. Further, the convergence model is simplified using the Multi-Stage Diffusion.

$$
\mathrm{CO}_{e}:=\mathrm{COP}_{\Theta}\left(\mathrm{M}^{e-1}\right)
$$

\subsection{Training}

In order to train the model, we consider $M$ as the $2 \mathrm{D}$ convolution matrix, here the blur kernels are drawn randomly. Moreover, for the deburring $I^{e}$ has the closed form solution:

$$
I^{e}=F T^{-1}\left(F T\left(\lambda M^{E} I_{2}+\varphi^{e} C O^{e}\right)\left(F T\left(\lambda M^{E} M+\varphi^{e}\right)\right)^{-1}\right)
$$

$F^{-1}$ In addition, $F$ is the inverse fourier transform and fourier transform. Moreover, the loss function is the negative PSNR of the de blurred image, the loss function gradient with respect to the parameters is given in the $\vartheta=\left\{\Gamma_{T}, \Theta\right\}$, here $\mathrm{T}$ indicates the task. Furthermore, the training scheme has been designed in such a way that it should be more effective. Here at first the iteration is set for the single and two parameters are trained in $\operatorname{COP}_{\Theta}$, these two parameters include $\Theta, \Gamma$. Later the iteration is increased and every time the result is refined from the previous step.

\section{RESULTS AND DISCUSSION}

In this section of research, we evaluate the CCM (constraint conditional model) through comparing our model with the other research techniques; moreover, this section is parted into the different sub-section. Furthermore, to evaluate the CCM we have considered the ideal computation environment of Intel i5 processor on windows 10 platform packed with 8 GB RAM and 2 GB NVidia graphics. Moreover, MATLAB has been used as the programming language to evaluate the CCM model.

\subsection{Dataset}

In this research work constraint conditional model is evaluated on three standard datasets i.e. Levin [20], Kohler [21] and Sun [22], these datasets are considered challenging as well as standard dataset. Moreover, Levin is the grey scale dataset and the entire 8-blur kernel image is depicted in the Figure 1. Figure 1 consists of eight figures from a) to h) i.e. BK1 to BK8 respectively. Similarly Figure 2 and Figure 3 presents the results on eight different kernel with different images. 


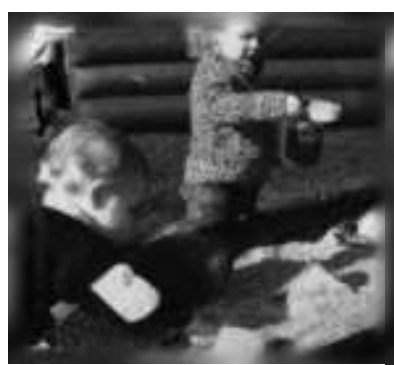

(a)

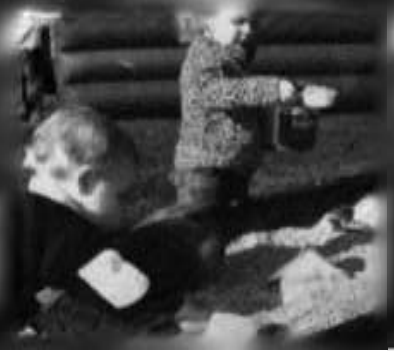

(b)

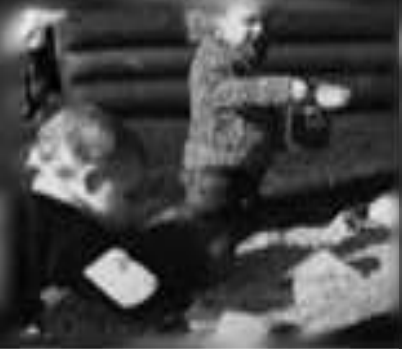

(c)

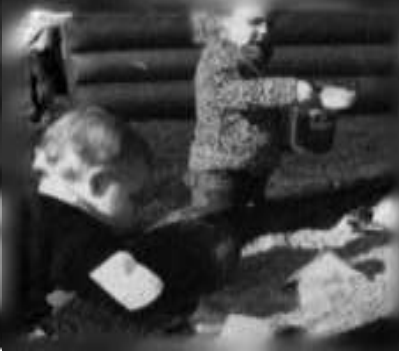

(d)

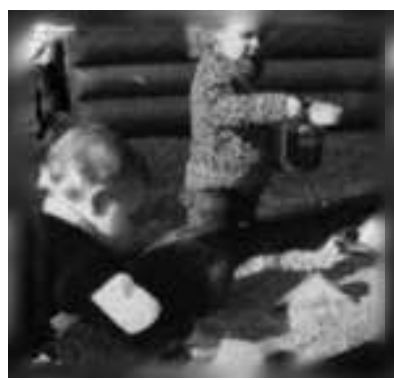

(e)

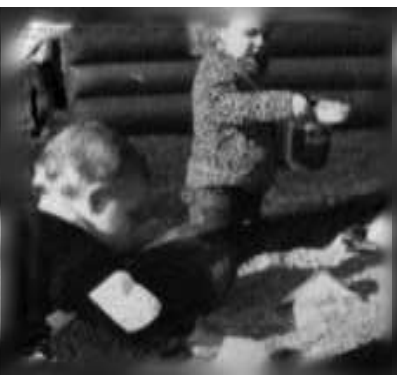

(f)

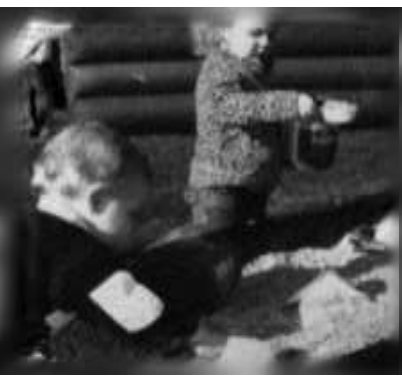

(g)

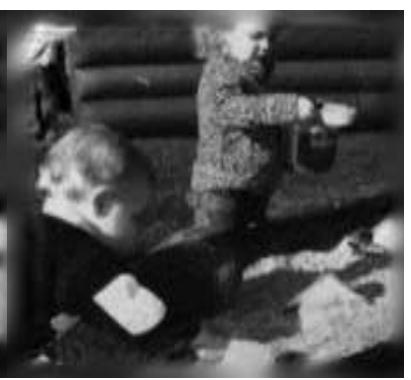

(h)

Figure 1. Results on eight different blur kernel on Levin (a) Blur Kernel 1, (b) Blur Kernel 2, (c) Blur Kernel 3, (d) Blur Kernel 4, (e) Blur Kernel 5, (f) Blur Kernel 6, (g) Blur Kernel 7, (h) Blur Kernel 8 [20]

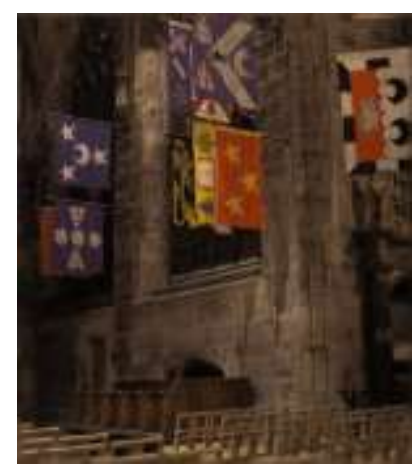

(a)

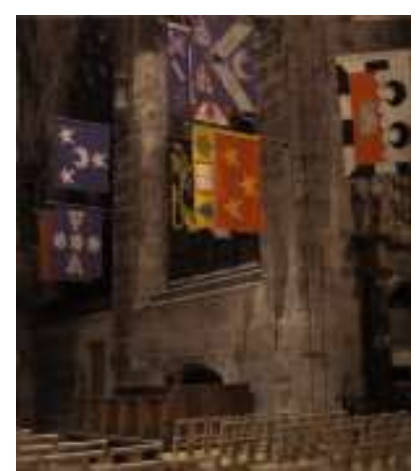

(e)

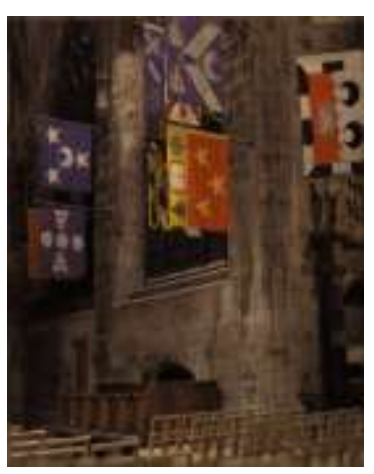

(b)

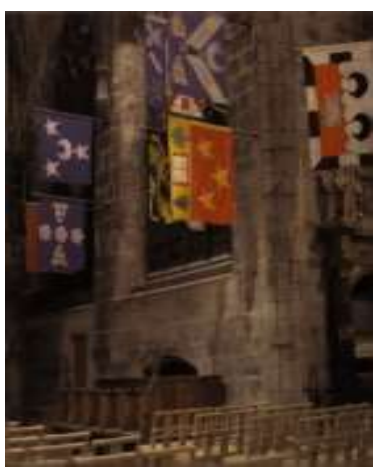

(f)

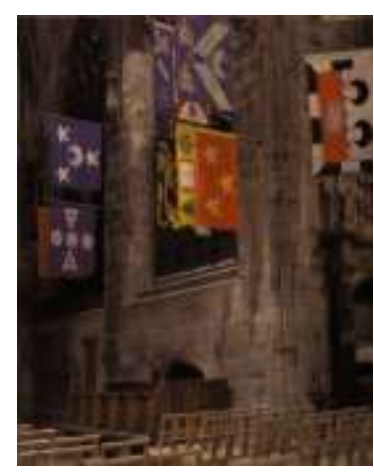

(c)

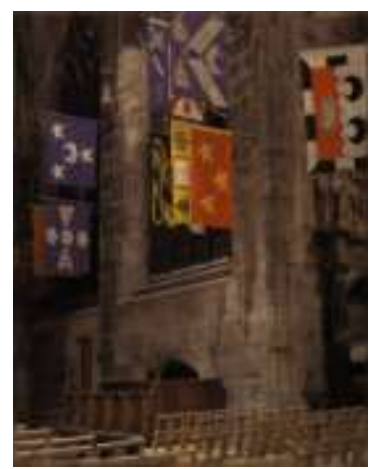

(g)

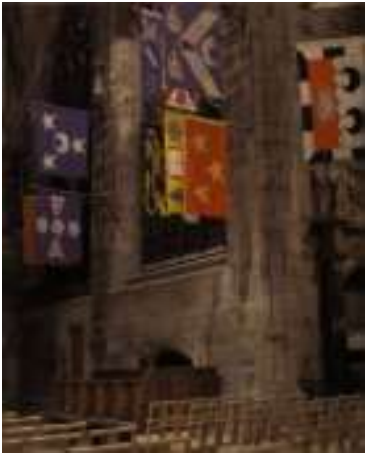

(d)

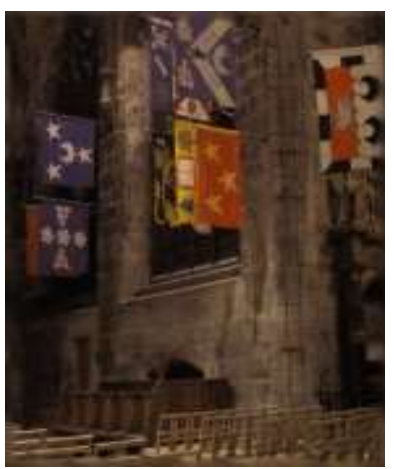

(h)

Figure 2. Results on eight different kernel on Kohler dataset (a) Blur Kernel 1, (b) Blur Kernel 2, (c) Blur Kernel 3, (d) Blur Kernel 4, (e) Blur Kernel 5, (f) Blur Kernel 6, (g) Blur Kernel 7, (h) Blur Kernel 8 [21] 


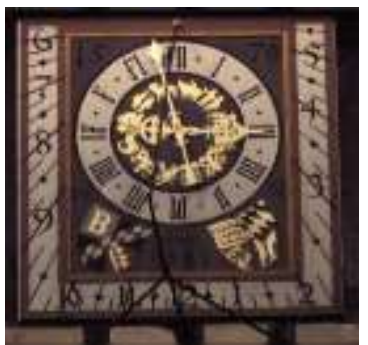

(a)

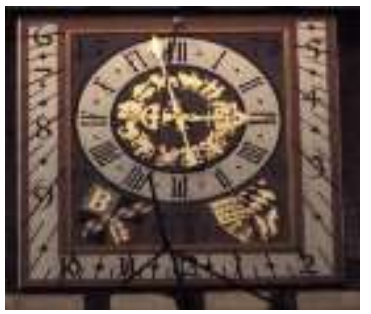

(e)

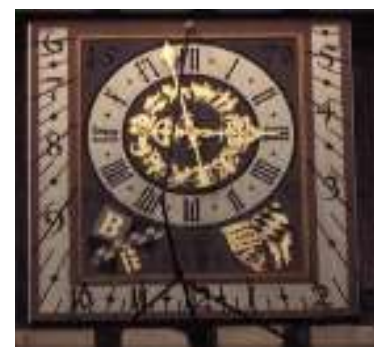

(b)

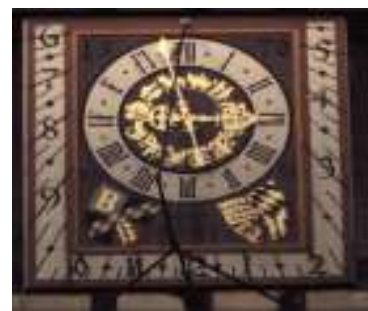

(f)

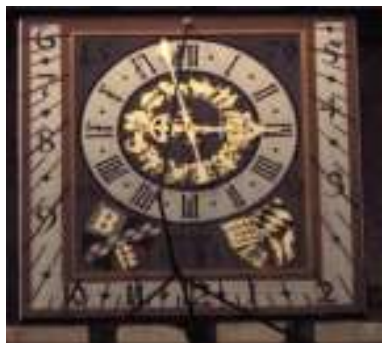

(c)

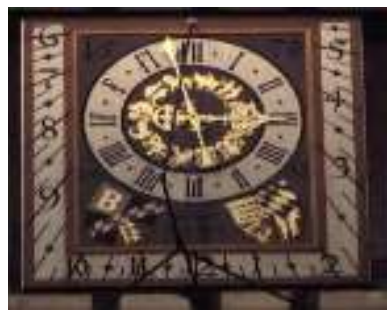

(g)

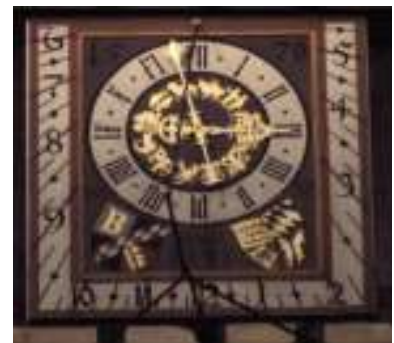

(d)

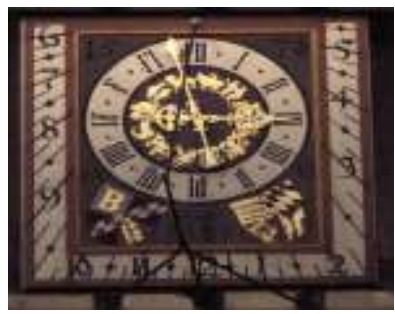

(h)

Figure 3. Results on eight different kernel on Kohler dataset (a) Blur Kernel 1, (b) Blur Kernel 2, (c) Blur Kernel 3, (d) Blur Kernel 4, (e) Blur Kernel 5, (f) Blur Kernel 6, (g) Blur Kernel 7, (h) Blur Kernel 8 [21]

\subsection{Image quality metrics comparison}

In an image processing, any processing implied to the given image causes the degradation in image quality. Moreover, to test the efficiency of CCM approach it is essential to perform similarity test, in this paper we have selected SSIM and PSNR as two image quality metrics which is used for determining how much quality is lost.

\subsubsection{PSNR (Peak Signal-to-noise Ratio)}

PSNR is the value observed in db (decibels) between the two-given image, here the ratio is considered for the original image and the reconstructed image, higher PSNR indicates the betterreconstructed image. PSNR represents the peak error image. Table 1 presents the comparison of various technique on the eight different kernel. In Table 1, first column presents the type of Blur kernel, which is placed in respective order of BK1 to BK 8. Second, third, fourth and fifth column represents the various state of art technique sixth column presents the PSNR value of existing protocol. Last column presents PSNR value of proposed model i.e. CCM (constraint condition model). Moreover, it is observed that every other model possesses the PSNR model below 30; existing model achieves the 30.235 whereas our model achieves the value of 34.383 in case of BK1. In case of BK2, our method achieves the PSNR value of 36.615 when compared to the existing value of 30.56, in case of BK3 CCM achieves 35.63 in comparison with existing model of 28.55. In case of BK4, CCM achieves the value of 35.93 when compared to the existing model of 22.652; BK5 of CCM achieves the value of 34.814 when compared to the existing value of 31.397 while other method remains on the lower side. Similarly, BK6, BK7 and BK8 achieves the PSNR value of 35.536, 33.631, 34.190 respectively in comparison with the existing model value of PSNR 21.073, 23.144 and 23.687 value respectively.

Table 1. Mean PSNR comparasion with various state-of-art technique

\begin{tabular}{ccccccc}
\hline Type of Blur Kernel & krishnan et.al. [23] & pan et.al.[9] & pan et.al.[8] & xu.et.al.[24] & tang et.al.[25] & CCM \\
\hline BK1 & 21.316 & 29.9131 & 27.841 & 26.293 & 30.235 & 34.3831 \\
BK2 & 20.292 & 29.8022 & 27.877 & 27.302 & 30.562 & 36.615 \\
BK3 & 22.171 & 30.0292 & 26.758 & 24.287 & 28.550 & 35.6320 \\
BK4 & 18.313 & 21.652 & 21.241 & 20.363 & 22.652 & 35.9360 \\
BK5 & 22.754 & 30.802 & 29.264 & 28.674 & 31.397 & 34.8149 \\
BK6 & 17.187 & 19.793 & 19.387 & 19.215 & 21.073 & 35.526 \\
BK7 & 18.504 & 22.155 & 21.601 & 21.092 & 23.144 & 33.631 \\
BK8 & 19.256 & 22.535 & 22.541 & 21.969 & 23.687 & 34.190 \\
\hline
\end{tabular}




\subsubsection{SSIM (structural similarity index)}

Structural Similarity index is the method for perception-based model and it considers the image degradation as the given perceived change in any structural information. Moreover, it also incorporates phenomena such as contrast masking and luminance masking. Table 2 represents the comparison of various technique along with the existing model. Moreover, comparison has been done by considering the 8-blur kernel; meanwhile the higher value of SSIM indicates the model efficiency is higher. In case of BK1 and BK2 our proposed model achieves the value of 86.39 and 91.66 when compared to the existing model 85.77 and 91.66 respectively, whereas other model remains on the lower side. In case of BK3, BK4 and BK5 existing model achieves the SSIM value of 82.21 and 58.07 and 88.01 whereas CCM model achieves 90.20, 89.29 and 89.29 respectively. Similarly, in case of BK5, BK 6 and BK7, the existing model achieves SSIM value of 47.01, 59.08, and 64.78 whereas CCM model achieves value of 89.29, 90.48 and 84.99 respectively. In case of BK8, existing model achieves PSNR value of 64.78 and CCM achieves 88.57. Moreover, through the comparison analysis it can be observed that CCM achieves the higher SSIM value for all eight kernel.

Table 2. Mean SSIM comparasion with various state-of-art technique

\begin{tabular}{ccccccccc}
\hline Blur Kernel Type & $\begin{array}{c}\text { Blur } \\
\text { Kernel 1 }\end{array}$ & $\begin{array}{c}\text { Blur } \\
\text { Kernel 2 }\end{array}$ & $\begin{array}{c}\text { Blur } \\
\text { Kernel 3 }\end{array}$ & $\begin{array}{c}\text { Blur } \\
\text { Kernel 4 }\end{array}$ & $\begin{array}{c}\text { Blur } \\
\text { Kernel 5 }\end{array}$ & $\begin{array}{c}\text { Blur } \\
\text { Kernel 6 }\end{array}$ & $\begin{array}{c}\text { Blur } \\
\text { Kernel 7 }\end{array}$ & $\begin{array}{c}\text { Blur } \\
\text { Kernel 8 }\end{array}$ \\
\hline krishnan et.al. [23] & 66.27 & 59.61 & 70.81 & 45.84 & 74.71 & 39.51 & 46.38 & 51.74 \\
pan et.al.[9] & 82.34 & 84.13 & 76.26 & 54.86 & 87.07 & 44.95 & 57.27 & 62.49 \\
pan et.al.[8] & 81.96 & 82.49 & 79.1 & 54.62 & 86.67 & 44.88 & 54.04 & 60.38 \\
xu.et.al.[24] & 81.08 & 82.61 & 74.18 & 52.21 & 85.64 & 45.53 & 52.42 & 60.09 \\
tang et.al.[25] & 85.77 & 85.93 & 82.21 & 58.07 & 88.01 & 47.01 & 59.08 & 64.78 \\
Proposed (CCM) & 86.3957 & 91.6608 & 89.4607 & 90.2075 & 89.2936 & 90.4837 & 84.9932 & 88.5793 \\
\hline
\end{tabular}

\section{CONCLUSION}

In this paper, we have proposed an efficient technique towards the blind deblurring named CCM (constraint conditional model), the main aim of CCM is to restore the image in its original form; the best advantage of CCM is that it provides handsome tradeoff between the image quality and efficiency. Furthermore, CCM is evaluated considering the three standard dataset i.e. Levin, Kohler and Sun, we have shown the image of all the eight-blur kernel result and later the comparison analysis is performed considering the two image quality metrics PSNR and SSIM. Constraint conditional model achieves the higher value of PSNR and SSIM compared to other techniques; this indicates that our method is highly efficient and successful in deblurring and restoring the original image. In addition, the deblurring is the vast imageprocessing concept and is improvised day by day, considering the future work several parameters have to be considered along with the various dataset and further this research work can be apply in the field of patern recognition, for the purpose of detecting text from blurred and low resolution images.

\section{ACKNOWLEDGEMENTS}

We gratefully thank the Visvesvaraya Technological University, Jnana Sangama, Belagavi for financial support extended to this research work.

\section{REFERENCES}

[1] R. Liu, Z. Li and Jiaya Jia, "Image partial blur detection and classification," 2008 IEEE Conference on Computer Vision and Pattern Recognition, Anchorage, AK, pp. 1-8, 2008.

[2] K. Bahrami, A. C. Kot, L. Li and H. Li, "Blurred Image Splicing Localization by Exposing Blur Type Inconsistency," in IEEE Transactions on Information Forensics and Security, vol. 10, no. 5, pp. 999-1009, 2015.

[3] J. Gast, A. Sellent and S. Roth, "Parametric Object Motion from Blur," 2016 IEEE Conference on Computer Vision and Pattern Recognition (CVPR), Las Vegas, NV, pp. 1846-1854, 2016.

[4] M. Hirsch, C. J. Schuler, S. Harmeling and B. Schölkopf, "Fast removal of non-uniform camera shake," 2011 International Conference on Computer Vision, Barcelona, pp. 463-470, 2011.

[5] N. Joshi, R. Szeliski, and D. J. Kriegman, "PSF estimation using sharp edge prediction," in IEEE Conference on Computer Vision and Pattern Recognition, pp. 1-8, 2008.

[6] S. Cho and S. Lee, "Fast motion deblurring," ACM Transactions on Graphics, vol. 28, no. 5, pp. 1-8, 2009.

[7] L. Xu, S. Zheng, and J. Jia, "Unnatural 10 sparse representation for natural image deblurring," in IEEE Conference on Computer Vision and Pattern Recognition, pp. 1107-1114, 2013.

[8] J. Pan, Z. Hu, Z. Su, and M.-H. Yang, "L0-regularized intensity and gradient prior for deblurring text images and beyond," IEEE Transactions on Pattern Analysis and Machine Intelligence, vol. 39, no. 2, pp. 342-355, 2017.

[9] J. Pan, D. Sun, H. Pfister, and M. H. Yang, "Blind image deblurring using dark channel prior," in IEEE Conference on Computer Vision and Pattern Recognition, pp. 1628-1636, 2016. 
[10] K. He, J. Sun, and X. Tang, "Single image haze removal using dark channel prior," IEEE Transactions on Pattern Analysis and Machine Intelligence, vol. 33, no. 12, pp. 2341-2353, 2011.

[11] Y. Yan, W. Ren, Y. Guo, R. Wang, and X. Cao, "Image deblurring via extreme channels prior," in IEEE Conference on Computer Vision and Pattern Recognition, vol. 2, no. 5, pp. 4003-4011, 2017.

[12] R. Fergus R, et al., "Removing camera shake from a single photograph," ACMTrans Graph, vol. 25, no. 3, pp. 787794, 2006.

[13] Q. Shan, J. Jia, and A. Agarwala "High-quality motion deblurring from a single image," ACM Trans Graph vol. 27, no. 3, pp. 1-10, 2008.

[14] Pan, Jinshan, Z. Su, "Fast 10-regularized kernel estimation for robust motion deblurring," IEEE Signal Process Lett vol. 20 , no. 9 , pp. 841-844, 2013.

[15] Z. Hu, J. B. Huang, and M. H. Yang, "Single image deblurring with adaptive dictionary learning," In: Proceedings of IEEE international conference on image processing (ICIP), pp 1169-1172, 2010.

[16] W. Ren, et al., "Image deblurring via enhanced low-rank prior," IEEE Trans Image Process, vol. 25, no. 7, pp. 3426-3437, 2016.

[17] J. Pan J, et al., "Blind image deblurring using dark channel prior," In: Proceedings of IEEE conference on computer vision and pattern recognition (CVPR), 2016, pp. 1628-1636.

[18] W. Zhao, H. Lu, and D. Wang, "Multisensor image fusion and enhancement in spectral total variation domain," IEEE Trans Multimedia, vol. 20, no. 4, pp. 866-879, 2017.

[19] W. Zhao, H. Lu, and D. Wang, "Defocus Blur Detection via Multi-Stream Bottom-Top-Bottom Fully Convolutional Network," In: The IEEE conference on computer vision and pattern recognition (CVPR), pp 30803088, 2017.

[20] A. Levin, Y. Weiss, F. Durand, and W. T. Freeman, "Understanding and evaluating blind deconvolution algorithms," in Proc. IEEE Comput. Vis. Pattern Recognit. (CVPR), pp. 1964-1971, 2009.

[21] R. Köhler, M. Hirsch, B. Mohler, B. Schölkopf, and S. Harmeling, "Recording and playback of camera shake: Benchmarking blind deconvolution with a real-world database," in Proc. Eur. Conf. Comput. Vis. (ECCV), pp. 2740, 2012.

[22] L. Sun, S. Cho, J. Wang, and J. Hays, "Edge-based blur kernel estimation using patch priors," in Proc. IEEE Int. Conf. Comput. Photogr. (ICCP), pp. 18, 2013.

[23] D. Krishnan, T. Tay, and R. Fergus, "Blind deconvolution using a normalized sparsity measure," in Proc. IEEE Comput. Vis. Pattern Recognit. (CVPR), pp. 233-240, 2011.

[24] L. Xu and J. Jia, "Two-phase kernel estimation for robust motion deblurring," in Proc. 11th Eur. Conf. Comput. Vis. (ECCV), pp. 157-170, 2010.

[25] S. Tang et al., "Multi-Regularization-Constrained Blur Kernel Estimation Method for Blind Motion Deblurring," in IEEE Access, vol. 7, pp. 5296-5311, 2019.

\section{BIOGRAPHIES OF AUTHORS}

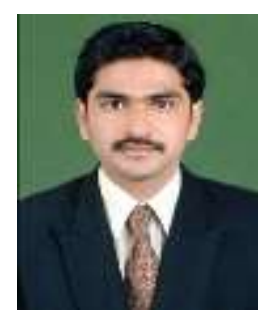

Ravikumar.H.C is a research scholar in the Department of Electronics and Communication Engineering at KSSEM, Bengaluru, affiliated to VTU, Belagavi and currently working as Asst. professor in Dept. of ECE at Ghousia College of Engineering, Ramanagaram. He has done his masters in VLSI design and embedded systems from VTU Extension Centre, PESCE, Mandya. He is a life member of IEI. His areas of interest are image processing, machine learning, pattern recognition and multimedia concepts.

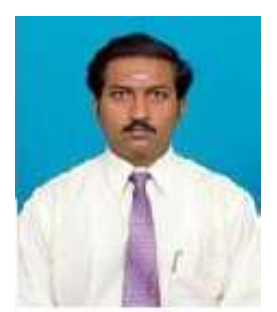

P. Karthik received Doctoral degree from Dr MGR University, Master from Sathyabama University, during the year 2013 and 2006. Currently he is working as a Professor in the Dept. of Electronics \& Communication Engineering at K S School of Engineering, Bengaluru. His current research interests are Acoustics sensors, image processing, machine learning, smart grids and mobile communication. He is actively involved in various professional bodies like, IEEE senior member, Member in IEI and Member in ISTE and IAENG and ACM. 